question arises. Why are not all such $\gamma$-rays observed in all processes involving high energy protons? The answer is not clear.

A fairly sound test of the hypothesis is obvious. Photon production should endure in some cases as long as positive electron emission does.

The considerations of this article undergo a slight modification if one chooses to introduce the notion of a light neutron such as that proposed by Pauli several years ago. This particle for which the name "neutret" is proposed (rather than the Italian form neutrino often used) ought to have Fermi statistics and a mass of the order of that of the electron. As mentioned in recent literature these properties would explain away the difficulties in transitions from the proton to the neutron as well as those of continuous $\beta$-ray spectra. In fact, it would follow that all $\beta$-ray spectra, positive as well as negative, would be continuous.

The excitation of the proton would presumably involve the ejection or annihilation of the neutret. The second alternative might correspond to the production of a negative proton from the neutron. The excited proton would definitely then be a Bose particle and would endure until annihilation or disintegration of the positive electron occurred. The energy available for the photons of the
Lea effect or the $\beta^{+}$emission might be less than that already mentioned by the mass and the binding energy of the neutret. This, however, is not serious.

One of the attractive features of the neutret hypothesis is the possibility of building an energetically stable proton with its help. This would require a mass at least as great as the difference between the proton and the sum of a neutron and a positive electron.

Note added March 15. The best available method for estimating the mass of the neutret is probably from the recent experiments in this laboratory where proton bombardment yielded positive electrons with energies up to at least $1.5 \times 10^{6}$ volts. The mass-equation gives $p-n$ $-\beta^{+}+\Delta E$ for the mass of the neutret where $p, n, \beta^{+}$stand for the masses of the proton, neutron and positive electron and $\Delta E$ is the difference between the threshold energy for the incident protons and the kinetic energy of the products. Numerical values show that the rest mass of the neutret is probably smaller than that of the electron. This is as F. Perrin (Comptes Rendus 197, 1625 (1933)) has already pointed out in good agreement with the distribution of energy in beta-ray disintegrations.

California Institute of Technology,

February 20, 1934.

\section{R. M. LANGER}

\title{
The Vibrational Spectrum of Water Vapor
}

Recently R. Mecke ${ }^{1}$ has published a rather complete analysis of seventeen of the vibration rotation bands of water vapor, together with an assignment of the vibrational quantum numbers. This work provides accurate values for the centers of most of the bands, though a few in the visible region of the spectrum seem for several reasons somewhat doubtful. With such complete information available, it seemed desirable to carry out a theoretical investigation of a nonlinear, symmetric triatomic molecule, similar to the treatment used by Adel and Dennison ${ }^{2}$ on $\mathrm{CO}_{2}$, and to apply the results to $\mathrm{H}_{2} \mathrm{O}$. This has been done by using first and second order perturbation theory, and it has been found that the vibrational energies of this type of molecule may be expected, to the degree of approximation involved, to obey a formula of the form

$$
\begin{aligned}
W=x_{1} V_{1}+x_{2} V_{2}+x_{3} V_{3} & +x_{11} V_{1}^{2}+x_{22} V_{2}^{2}+x_{33} V_{3}^{2} \\
& +x_{12} V_{1} V_{2}+x_{13} V_{3} V_{3}+x_{23} V_{2} V_{3}+x_{0},
\end{aligned}
$$

where the $V$ 's are the vibrational quantum numbers, and the $x$ 's are coefficients which may be determined from the experimentally observed positions of the bands. Nine of the known bands are necessary in determining the constants, and the remainder may be used to check the validity of the equation. The check here is satisfactory for all but two bands, and the discrepancy for these is probably due to uncertainty in the positions of the band centers.

The theoretical treatment gives these $x$ 's in terms of the true mechanical frequencies of vibration of the molecule for infinitesimal amplitudes, of the interatomic distances, and of the constants in the assumed potential function. These expressions may be solved at once for the mechanical frequencies, which are necessary for the evaluation of the zeroth order binding constants, giving the results:

$$
\begin{aligned}
& \omega_{1}=x_{1}-x_{11}-\frac{1}{2} x_{12}-\frac{1}{2} x_{13}, \\
& \omega_{2}=x_{2}-x_{22}-\frac{1}{2} x_{12}-\frac{1}{2} x_{23}, \\
& \omega_{3}=x_{3}-x_{33}-\frac{1}{2} x_{13}-\frac{1}{2} x_{23} .
\end{aligned}
$$

For water the numerical values of the coefficients $x_{i j}$ are as follows:

$$
\begin{array}{lll}
x_{1}=3796.0 \mathrm{~cm}^{-1} & x_{11}=-39.5 \mathrm{~cm}^{-1} & x_{12}=-106.1 \mathrm{~cm}^{-1}, \\
x_{2}=3674.8 \mathrm{~cm}^{-1} & x_{22}=-70.2 \mathrm{~cm}^{-1} & x_{13}=-21.0 \mathrm{~cm}^{-1}, \\
x_{3}=1615.0 \mathrm{~cm}^{-1} & x_{33}=-19.5 \mathrm{~cm}^{-1} & x_{23}=-18.9 \mathrm{~cm}^{-1} .
\end{array}
$$

Calculating from these the values of the $\omega$ 's, these results are obtained:

$$
\omega_{1}=3899.0 \mathrm{~cm}^{-1}, \quad \omega_{2}=3807.5 \mathrm{~cm}^{-1}, \quad \omega_{3}=1654.5 \mathrm{~cm}^{-1} .
$$

It is interesting to note that one of these, $\omega_{1}$, differs by as much as 150 wave numbers from the observed position of the fundamental.

It is hoped before long to publish this treatment in more detail and to give the values of the constants appearing in the potential function used.

California Institute of Technology, March 13, 1934.

${ }^{1}$ R. Mecke et al., Zeits. f. Physik 81, 313, 445, 465 (1933). ${ }^{2}$ A. Adel and D. M. Dennison, Phys. Rev. 43, 716 (1933). 